\title{
Analysis of Single Cell Curved Box Girder Bases on Finite Strip Element Theory
}

\author{
Wang Feng ${ }^{1,2, a, *}$, Zhang Jian $^{3, b}$ and Jiang Lei ${ }^{3, c}$ \\ ${ }^{1}$ Institute of Bridge Engineering, Tongji University, Shanghai, China \\ ${ }^{2}$ Jiangsu Yangtze River Bridge Co., Ltd., Nanjing, China \\ ${ }^{3}$ Nanjing University of Aeronautics and Astronautics, Nanjing, China \\ a wangfjydq@163.com, ${ }^{b}$ jianzhang78@126.com, c leijnuaa@163.com \\ *Wang Feng
}

Keywords: single cell curved box girder; finite strip element theory; mechanical analysis.

\begin{abstract}
Finite strip element theory is applied to the analysis of single cell curved box girder. Firstly finite strip element theory is introduced and stiff matrix of the finite curved strip is deduced. The problem of the element coupling of strip element stiff matrix by using the orthogonal property of harmonious function and the problem of numerical integration of the element are solved. The equilibrium equation is deduced in detail. Through analysis of a classic example, some useful conclusions of applying finite strip element theory to analysis of curved box girder are obtained.
\end{abstract}

\section{Introduction}

Box cutting plane has been widely applied in practical engineering for its own merits such as excellent torsion property and slight weight. With the development of civil engineering and bridge engineering and with the advance of construction technology, curve box girder is used more and more widely[1]. As for continuous curve box, it is much complex to complete spatial analysis precisely[2-4]. However, finite curve strip method can satisfy the stability and precision conditions and at the same time the computing parameter which is necessary to be input are few, which makes finite curve strip method become the efficient computational method[5-6]. Finite curve strip method is a special form of the finite element method whose physical parameters can be determined easily[7]. Thus, it is necessary in do some research work in structural analysis theory of curved box girder bridge. In structural analysis theory of curved box girder bridges, finite curve strip method is an expedient and applied method. But by now, the research results by this method are rare.

Thus in this paper, directed against single cell curved box girder bridges, mechanical analysis is studied by applying Finite strip element theory. Based on finite strip element theory, stiffness matrix of finite curved strip element is deduced and then mechanical analysis of the single cell curved box girder bridge is completed.

\section{Finite Strip Element Theory}

Finite strip element theory utilizes the different kinds of $\varphi$, called conic angle, to depict different relations between strain and displacement(Figure 1). If $\varphi=0^{\circ}$, the shell is a pole shell; if $\varphi=90^{\circ}$, it is sector; if $0^{\circ}<\varphi<90^{\circ}$, it is a conic shell whose top part is cut off. The equation of depicting relation between strain and displacement based on finite strip element theory is as follows:

$$
\begin{aligned}
& \varepsilon_{x}=\frac{\partial u}{\partial x}, \quad \varepsilon_{\theta}=\frac{1}{r} \frac{\partial v}{\partial \theta}+\frac{w \cos \varphi+u \sin \varphi}{r}, \quad \gamma_{x \theta}=\frac{1}{r} \frac{\partial u}{\partial \theta}+\frac{\partial v}{\partial x}-\frac{v \sin \varphi}{r} \\
& \mathrm{X}_{x}=-\frac{\partial^{2} w}{\partial x^{2}}, \quad \mathrm{X}_{\theta}=-\frac{1}{r^{2}} \frac{\partial^{2} w}{\partial \theta^{2}}+\frac{\cos \varphi}{r^{2}} \frac{\partial v}{\partial \theta}-\frac{\sin \varphi}{r} \frac{\partial w}{\partial x} \\
& \mathrm{X}_{x \theta}=2\left(-\frac{1}{r} \frac{\partial^{2} w}{\partial x \partial \theta}+\frac{\sin \varphi}{r^{2}} \frac{\partial w}{\partial \theta}+\frac{\cos \varphi}{r} \frac{\partial v}{\partial x}-\frac{\sin \varphi \cos \varphi}{r^{2}} v\right)
\end{aligned}
$$




\section{Stiffness Matrix of Finite Curved Strip Element}

\subsection{Deduction of Stiffness Matrix}

As for pinned curve box structure, displacement interposition function of curve strip element (Figure 2) is:

$$
f=\left\{\begin{array}{l}
u \\
v \\
w
\end{array}\right\}=\sum_{m=1}^{r}\left\{\begin{array}{l}
u_{m} \\
v_{m} \\
w_{m}
\end{array}\right\}=\sum_{m=1}^{r}[\mathbf{N}]_{m}\left\{\delta_{m}\right\}
$$

$u_{m}, v_{m}, w_{m}$ in $\mathrm{Eq}(2)$ are respectively the $m$ th item of displacement of $x, y, z$ directions. [ $\mathbf{N}_{m}$ ] is the function as follows:

$$
[\mathbf{N}]_{m}=\left[\begin{array}{rccccccc}
N_{1 m} & 0 & 0 & 0 & N_{2 m} & 0 & 0 & 0 \\
0 & N_{3 m} & 0 & 0 & 0 & N_{4 m} & 0 & 0 \\
0 & 0 & N_{5 m} & N_{6 m} & 0 & 0 & N_{7 m} & N_{8 m}
\end{array}\right]
$$

Where $N_{1 m}=\left(1-\frac{x}{b}\right) Y_{m}, N_{2 m}=\frac{x}{b} Y_{m}, N_{3 m}=\left(1-\frac{x}{b}\right) \frac{Y_{m}^{\prime}}{K_{m}}, N_{4 m}=\frac{x}{b} \frac{Y_{m}^{\prime}}{K_{m}}, N_{5 m}=\left(1-\frac{3 x^{2}}{b^{2}}+\frac{2 x^{3}}{b^{3}}\right) Y_{m}$, $N_{6 m}=\left(x-\frac{2 x^{2}}{b}+\frac{x^{3}}{b^{2}}\right) Y_{m} \quad, \quad N_{7 m}=\left(\frac{3 x^{2}}{b^{2}}-\frac{2 x^{3}}{b^{3}}\right) Y_{m} \quad, \quad N_{8 m}=\left(-\frac{x^{2}}{b}+\frac{x^{3}}{b^{2}}\right) Y_{m}$ $\left\{\delta_{m}\right\}=\left[u_{i}, v_{i}, w_{i}, \theta_{y i}, u_{j}, v_{j}, w_{j}, \theta_{y j}\right]_{m}^{T} . Y_{m}$ is a harmonious function and when the boundary condition is pinned, $Y_{m}$ is expressed as follows:

$$
Y_{m}=\sin K_{m} \theta
$$

Where $K_{m}=\frac{m \pi}{\alpha}$ and $\alpha$ is the angle as shown in Figure 1. $\theta$ is the angular coordination and $b$ is the width of the strip element.

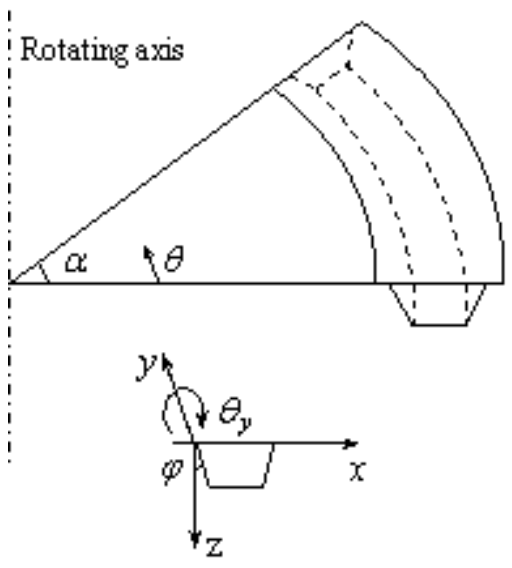

Figure 1 Curve box structure.

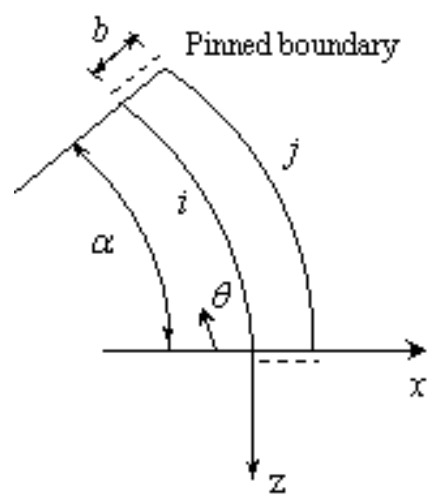

Figure 2 Curve strip element. 
Based on theory of elasticity, the stiff matrix of curve strip element can be expressed as follows:

$$
\begin{aligned}
\mathbf{K}_{e} & =\int_{V} \mathbf{B}^{T} \mathbf{D B} d V=\int_{V}\left[\mathbf{B}_{1} \mathbf{B}_{2} \cdots \mathbf{B}_{r}\right]^{T} \mathbf{D}\left[\mathbf{B}_{1} \mathbf{B}_{2} \cdots \mathbf{B}_{r}\right] d V \\
& =\int_{V}\left[\begin{array}{ll}
\mathbf{B}_{1}^{T} \mathbf{D B}_{1} & \mathbf{B}_{1}^{T} \mathbf{D B}_{2} \cdots \mathbf{B}_{1}^{T} \mathbf{D B}_{r} \\
\mathbf{B}_{2}^{T} \mathbf{D B}_{1} & \mathbf{B}_{2}^{T} \mathbf{D B}_{2} \cdots \mathbf{B}_{2}^{T} \mathbf{D} \mathbf{B}_{r} \\
\cdots & \\
\mathbf{B}_{r}^{T} \mathbf{D B}_{1} & \mathbf{B}_{r}^{T} \mathbf{D B}_{2} \cdots \mathbf{B}_{r}^{T} \mathbf{D B}_{r}
\end{array}\right] d V=\left[\begin{array}{ll}
\mathbf{K}_{e 11} & \mathbf{K}_{e 12} \cdots \mathbf{K}_{e 1 r} \\
\mathbf{K}_{e 21} & \mathbf{K}_{e 22} \cdots \mathbf{K}_{e 2 r} \\
\cdots & \\
\mathbf{K}_{e r 1} & \mathbf{K}_{e_{r 2}} \cdots \mathbf{K}_{e r r}
\end{array}\right]
\end{aligned}
$$

Where $\mathbf{D}$ is elastic matrix and strain matrix $\mathbf{B}$ can be derived by putting Eq.(2) into Finite strip element theory Eq.(1). In Eq.(5), we have:

$$
\mathbf{K}_{e m n}=\int_{V} \mathbf{B}_{m}^{T} \mathbf{D B}_{n} d V
$$

By using the orthogonal property of the function $Y_{m}=\sin \frac{m \pi}{\alpha} \theta$, Eq. (5) can be written as follows:

$$
\mathbf{K}_{e}=\operatorname{diag}\left[\mathbf{K}_{e 11} \quad \mathbf{K}_{e 22} \cdots \mathbf{K}_{e r r}\right]
$$

The $m$ th item of finite curve strip controlling equation of pinned curve box is:

$$
\mathbf{K}_{m m} \mathbf{U}_{m}=\mathbf{R}_{m}
$$

Where $\mathbf{K}_{m m}=\sum_{e} \mathbf{T}^{T} \mathbf{K}_{e m m} \mathbf{T} . \mathbf{T}$ is rotational matrix from total coordination system to local coordination system. $\mathbf{U}_{m}$ and $\mathbf{R}_{m}$ are respectively the $m$ th item of total displacement vector and total load vector. The pinned curve box displacement $\mathbf{U}$ is:

$$
\mathbf{U}=\sum_{m=1}^{r} \mathbf{U}_{m}
$$

\subsection{Numerical Integral of Element of Stiffness Matrix}

In Eq.(6), stiffness matrix $\left[\mathbf{K}_{e}\right]_{m m}$ of curved strip element can be determined by numerical integral method. Because of the uniform thickness of curved strip element, stiffness matrix $\left[\mathbf{K}_{e}\right]_{m m}$ can be written into the form of area integral of a cone, which is as follows:

$$
\mathbf{K}_{e m m}=\int_{0}^{\alpha} \int_{0}^{b}[\mathbf{B}]_{m}^{T}[\mathbf{D}][\mathbf{B}]_{m} r d x d \theta
$$

Where $r$ is the distance between the differential point of curved strip element and rotating axis of single cell curved box girder bridge and the distance $r$ can be written as follows:

$$
r=r(x)=r_{1}+x \sin \varphi
$$

Where $x$ is a variable depicting the variability of the width of curved strip element, which changes from 0 to $b ; r_{1}$ is the distance between the beginning point of curved strip element and rotating axis of single cell curved box girder bridge. From the deduction process, as is known that every element of functions $[\mathbf{B}]_{m}^{T}[\mathbf{D}][\mathbf{B}]_{m}$ has $\sin ^{2} K_{m} \theta$ or $\cos ^{2} K_{m} \theta$. And so, Eq.(10)becomes:

$$
\mathbf{K}_{e m m}=\frac{\alpha}{2} \int_{0}^{b}[\overline{\mathbf{B}}(x)]_{m}^{T}[\mathbf{D}][\overline{\mathbf{B}}(x)]_{m} r(x) d x
$$

Where matrix $\overline{\mathbf{B}}(x)$ is the corresponding result of matrix $\mathbf{B}(x)$ which does not contain $\sin K_{m} \theta$ and $\cos K_{m} \theta$. In order to make the numerical integral convenient and concise, the old coordinate system is diverted into $\xi$ coordinate system. Supposing $x=\frac{b}{2}(1+\xi)$ and $\xi \in[-1,1]$, we have $r=r_{1}+\frac{b}{2}(1+\xi) \sin \varphi$ and then Eq.(10) becomes:

$$
\mathbf{K}_{e}=\frac{\alpha b}{4} \int_{-1}^{1}[\overline{\mathbf{B}}(\xi)]_{m}^{T}[\mathbf{D}][\overline{\mathbf{B}}(\xi)]_{m} r(\xi) d \xi
$$

Eq.(13) can be easily calculated with Gaussian integral method. 


\section{Procedure Validation and Example Analysis}

In order to apply finite strip element-flexibility theory to the analysis of continuous curved box girder with diaphragms, the corresponding procedure called CBSFSM (in Fortran) is compiled in this paper. At first, validate the correctness of analytical procedure of pinned curved box girder which is used by CBSFSM. A model of pinned curved box girder is studied in this paper. The length of the middle axis is $L=120 \mathrm{~cm}$. The values of displacement parameters and the widths of the cutting plane are shown in Table 1. The curve strip element and the number of the line are shown in Figure 3. The plan of the pinned curved box girder is shown in Figure 4. he radius is $R=300 \mathrm{~cm}$ and the angle is $\alpha=0.4 \mathrm{rad}$. In order to compare with the results in Ref.[5] conveniently, the vertical loads $p_{1}=3.89 \mathrm{~N} / \mathrm{cm}$ and $p_{2}=7.78 \mathrm{~N} / \mathrm{cm}$ are applied to the curved box along the line on the cutting planes of number 2 and number 5. The series item $m$ is supposed to 25. The internal force $\sigma_{\theta}$ in the shear direction and the internal force $M_{r}$ in the radial direction are respectively shown in Figure 5 and Figure 6.

Table 1 True values of displacement parameters and the widths of the cutting plane

\begin{tabular}{cccccc}
\hline $\begin{array}{c}\text { Parameter's } \\
\text { name }\end{array}$ & $\begin{array}{c}\text { Young's } \\
\text { modulus }\end{array}$ & $\begin{array}{c}\text { Poisson's } \\
\text { ratio }\end{array}$ & $\begin{array}{c}\text { Thickness } \\
\text { of the plane } \\
\text { board }\end{array}$ & $\begin{array}{c}\text { Thickness } \\
\text { of bottom } \\
\text { board }\end{array}$ & $\begin{array}{c}\text { Thickness } \\
\text { of middle } \\
\text { board }\end{array}$ \\
\hline $\begin{array}{c}\text { Unit } \\
\text { Value }\end{array}$ & $\begin{array}{c}\mathrm{N} / \mathrm{cm}^{2} \\
280000\end{array}$ & 0.4 & $\mathrm{~cm}$ & 0.4 & 0.4 \\
\hline
\end{tabular}

Figure 3 Curve strip element and the number of the line $/ \mathrm{cm}$.

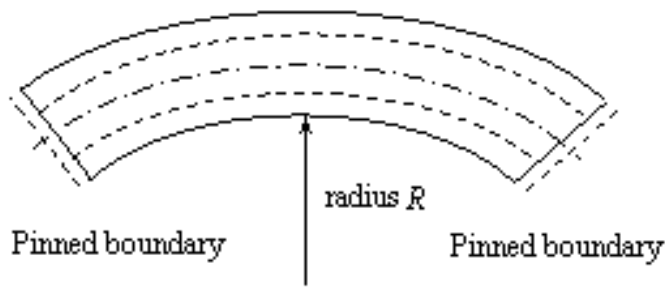

Figure 4 Applied loads of continuous curve box.

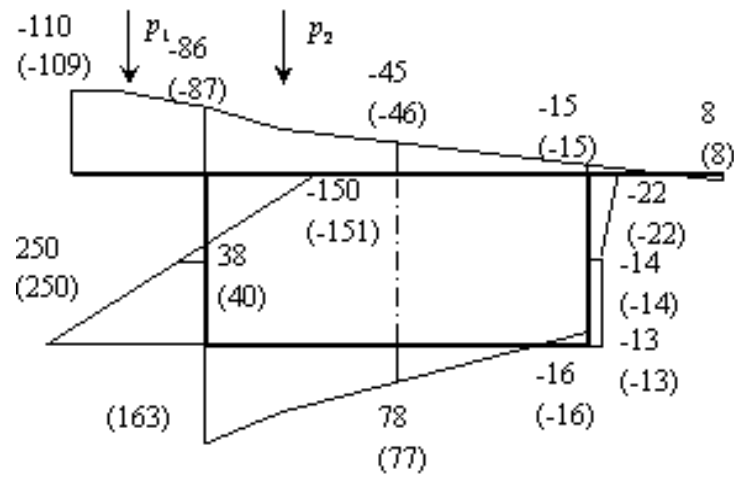

Figure 5 Internal force in the shear direction $/\left(\mathrm{N} / \mathrm{cm}^{2}\right)$. 


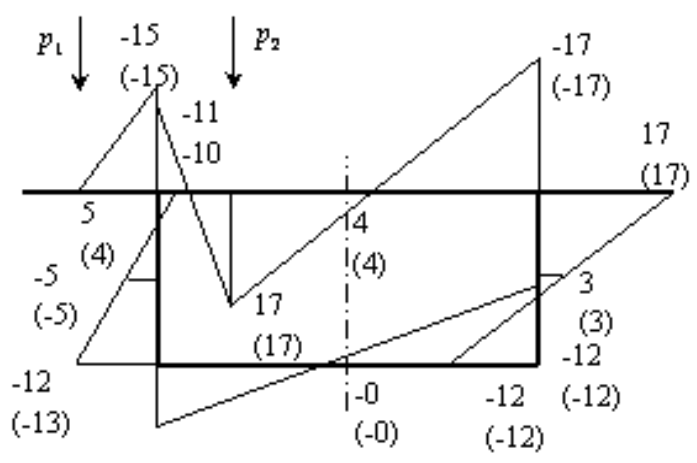

Figure 6 Internal force in the radial direction /( $\mathrm{N} \mathrm{cm})$.

The results in brackets in Figure 5 and Figure 6 are those in Ref.[5] and the results without brackets are gained from the analytical procedure of pinned curved box girder which is used by CBSFSM. Comparing the two kinds of results, the generalized stresses are very close to each other, which also indicates that the analytical procedure of pinned curved box girder is correct and reliable.

\section{Conclusion}

From finite strip element theory, a concise and reliable computational method for mechanical analysis of continuous curved box girder with diaphragm is studied in this paper, which needs fewer elements compared to other computational methods. The computational process is fast, correct, steady and convergent. The data of series item $m$ is important and the proper value can be determined by gradual comparison method. Finite strip element theory considers that plane effect and bending effect are irrelative while in fact, those two effects are interactional. Thus, much more precise and proper analytical theory for continuous curved box girder will be studied.

\section{References}

[1] Xiang H. F. (2001) Advanced theory of Bridge structure. Beijing: The Peoples' Communications Press.

[2] Xiao R. C. (2002) Analysis of bridge structure and program system. Beijing: The Peoples' Communications Press.

[3] Ghani R A, Hangang U. (1991) Single cell multicell box-girder finite element. Structure Engineering. ASCE, 117(10): 2953-2971.

[4] Zhao Z. M., Chen B C. (1997) Analysis of pole system and box girder bridge and program designing. Guangzhou: Huanan University of Science and Engineering Press.

[5] Zhang You Qi. (1978) Finite strip analysis of box girder bridge. Beijing: The Peoples' Communications Press.

[6] Shu S. H. (2003) Parameter stochastic back analysis method and object oriented programming design. Master Dissertation, Nanjing: Hohai University.

[7] Fan M. Y., Zhang Y. (1982) Basis of optimization technology. Beijing: Tsinghua University Press. 